\title{
REINGENIERÍA DE LOS BLOWERS DEL SISTEMA DE FRENADO DE UN CAMIÓN ELÉCTRICO DE 320 TONELADAS DE CARGA PARA MINERÍA EN EL ALTIPLANO PERUANO
}

\author{
REENGINEERING OF THE BLOWERS IN THE BRAKING SYSTEM OF A 320 \\ LOAD TONS ELECTRIC TRUCK FOR PERUVIAN MINING IN THE PLATEAU
}

\author{
Daniel R. Gutiérrez Ayala* \\ Ricardo J. Palomares Orihuela**
}

RECEPCIÓN: JUNIO DE 2017

ACEPTACIÓN: JULIO DE 2017

\section{RESUMEN}

La presente investigación comprende el rediseño del sistema de frenado de un camión eléctrico de 320 toneladas de carga a través de la modificación del sistema de enfriamiento de resistencias, que utiliza los actuadores llamados blowers en la aplicación de un retardo dinámico en la operación regular del sistema de frenado de los camiones de carga de alta minería, cuyas operaciones se realizan en altitudes por encima de los $4000 \mathrm{msnm}$. El estudio se basa en la alta incidencia de fallas de los sistemas eléctricos expuestos a condiciones ambientales extremas de temperatura, humedad y presión, por lo que se propone el desarrollo de la reingeniería del sistema motriz de los blowers a través del reemplazo de su motor eléctrico por un motor hidráulico de altas revoluciones.

Palabras clave: Blower, bomba hidráulica de engranajes, motor hidráulico, electroválvula.

\begin{abstract}
The present investigation includes the redesign of the braking system of an electric truck of 320 tons of load, through the modification of the system of resistance cooling, which uses the actuators called Blowers, in the application of a dynamic delay in the regular operation Of the braking system of high-load mining trucks, whose operations are carried out at altitudes above 4000 meters. The study is based in the detection of continuous failures of the electrical systems exposed to critical environmental conditions of temperature, humidity and pressure. I proceed to develop a thorough study of hydraulic engineering to replace the electric Blowers for hydraulic actuators by a high-revving engine Keywords: Blower, hydraulic gear pump, hydraulic motor, solenoid valve.
\end{abstract}

\footnotetext{
* Magíster en Dirección de la Producción y Mejora de Procesos Industriales de la Universidad de Barcelona y OBS, Ingeniero Mecatrónico de la Universidad Ricardo Palma, Especialista en Aseguramiento de Calidad del Product Support Group de la Empresa Komatsu Mitsui Maquinarias Perú S. A. <daniel.gutierrez@kmmp.com.pe>

** Magíster en Ciencias de la Educación con mención en Docencia Universitaria, Maestría en Ciencias de la Electrónica con mención en Ingeniería Biomédica, Doctorado en Ciencias de la Educación, Ingeniero Mecatrónico de la Universidad Nacional de Ingeniería, docente de las asignaturas de Robótica, Inteligencia Artificial, Mecatrónica Medica y Diseño Mecatrónico de la Escuela Profesional de Ingeniería Mecatrónica de la Universidad Ricardo Palma. <rpalomares@ieee.org>
} 


\section{Introducción}

La minería es una de las principales fuentes de riqueza del Perú. Existen múltiples yacimientos cuyo potencial ha sido catalogado como el cuarto más grande del mundo. Actualmente, nuestro país ocupa el puesto 7 dentro de los 10 principales países productores de minerales, con un valor de producción estimado en 27,063 millones de dólares. La minería peruana tiene como principal ventaja competitiva la diversificación de sus minerales. En sus principales yacimientos, se produce cobre, oro, plata, plomo, zinc, estaño, molibdeno, hierro, cadmio, mercurio, selenio, indio, entre otros metales. El Perú es el primer productor en la región de zinc, estaño, plomo y oro; y ocupa el segundo lugar en la producción de cobre, plata, molibdeno, selenio, cadmio y roca fosfórica [1]. La inversión destinada al sector minero ha tenido una tendencia creciente durante los últimos tres quinquenios, como se muestra en la figura 1.

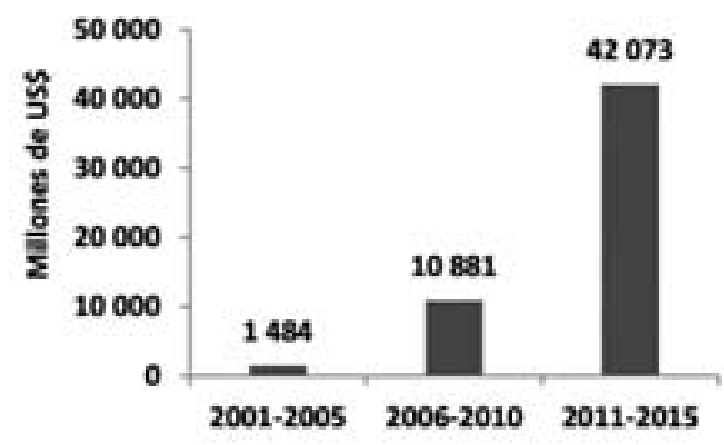

Fig. 1. Evolución de inversiones en sector minero.

Fuente: Reporte de Análisis económico sectorial - Osinergmin 2016.

Dentro de los procesos de preparación de la materia prima, se encuentran los de extracción y transporte de material. Los camiones eléctricos (ver figura 2) han ingresado en el mercado peruano como medios de transporte confiable y de alto rendimiento de materias primas y desmonte de materiales en minas. Los camiones representan el grupo más importante de los equipos de minería a nivel mundial dada su función, costo y cantidad.

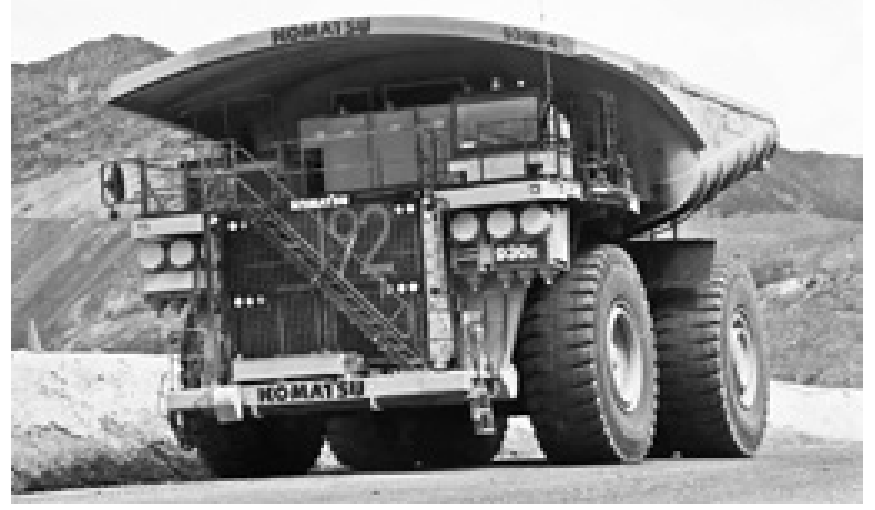

Fig. 2. Camión minero eléctrico en transporte de minerales.

Fuente: http://ims.infomine.com/265101/image.jpeg 
El principal problema que se presenta en los camiones mineros de alto tonelaje es la falla en uno de sus sistemas de frenado y control de velocidad. Los blowers, que son los ventiladores y actuadores principales en la disipación de la energía calorífica de las resistencias del sistema de frenado de retardo, fallan prematuramente cuando llegan alrededor del 30\% de su vida útil. Estas fallas son propiamente eléctricas y generan detenimiento de equipos, impacto en paradas de producción, costos en mantenimientos, entre otros. En ese sentido, es importante el estudio y mejora de fallas de equipos de uso masivo, dado que el impacto en la minería contempla beneficios de mayor envergadura, de allí que esta sea una de las principales razones por las cuales se elige como motivo de investigación al camión minero.

\section{Diseño y reingeniería de los sistemas}

En esta sección, se realiza el rediseño del sistema actual de frenado por retardo utilizado por los actuadores blower. Debido a las repetitivas fallas ocurridas durante su funcionamiento en el sistema motriz eléctrico de los blowers, se decide aplicar una reingeniería mediante una propuesta del tipo hidráulicomecánico y eléctrico.

El motor soplador o blower es una unidad modelo 5GY19AL6 (ver figura 3), de corriente continua, de cuatro polos de conmutación en serie, y que está montado dentro de la caja de los conjuntos de resistencias de retardo. Tiene montado dos ventiladores, uno en cada extremo del eje de la armadura, la cual suministra aire para disipar el calor del conjunto de resistencias durante la aplicación del retardo dinámico [2].

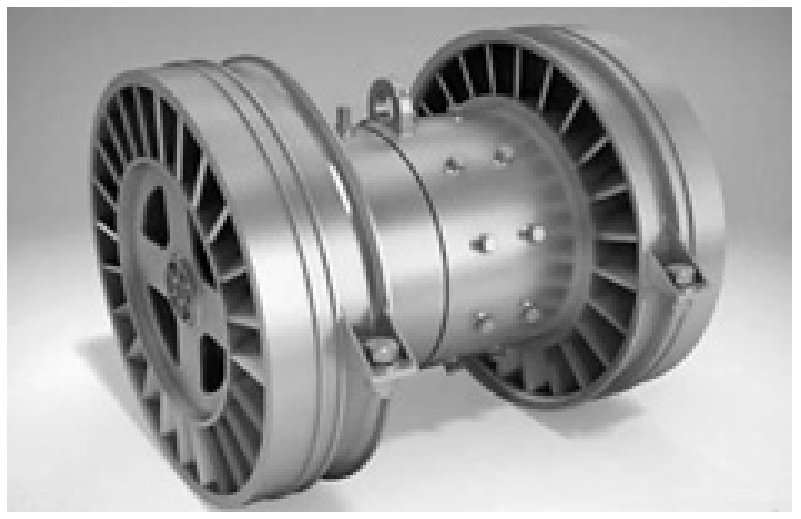

Fig. 3. Blower del sistema de frenado.

Fuente: Anglo American 2007 Neuralis Capacitación.

Se opta, por lo tanto, de dos modificaciones de los sistemas generales del funcionamiento del camión eléctrico: modificación al sistema hidráulico y al sistema eléctrico. Luego, se diseñan los componentes mecánicos menores para la implementación del sistema y, finalmente, se generará un kit de implementación.

\subsection{Estructura mecánica}

A continuación, se presentan las características del blower utilizado en camiones de 320 TN, que incluyen la estructura, dimensionado y especificaciones técnicas para la reingeniería del mismo. 


\section{Blower o motor soplador}

- Modelo: 5GY19A5, A6, E2, E3, L1, L2, L3, L4, L5, L6

- Clasificación: 4-polos, polo-conmutador, Series-Wound, Motor DC

- Rotación: velocidad máxima permisible (rpm): 3600

- $\quad$ Rango nominal: $65 \mathrm{HP}, 325 \mathrm{~V}$ at $3500 \mathrm{rpm}$

- Armadura: 0.0343

- Campo de excitación y conmutación: 0.0215

La estructura mecánica del blower consta de siete partes: rodamientos, caja de rodamiento, head frame, ventiladores, caja de cobertura, sellos y tapas (ver figura 4).

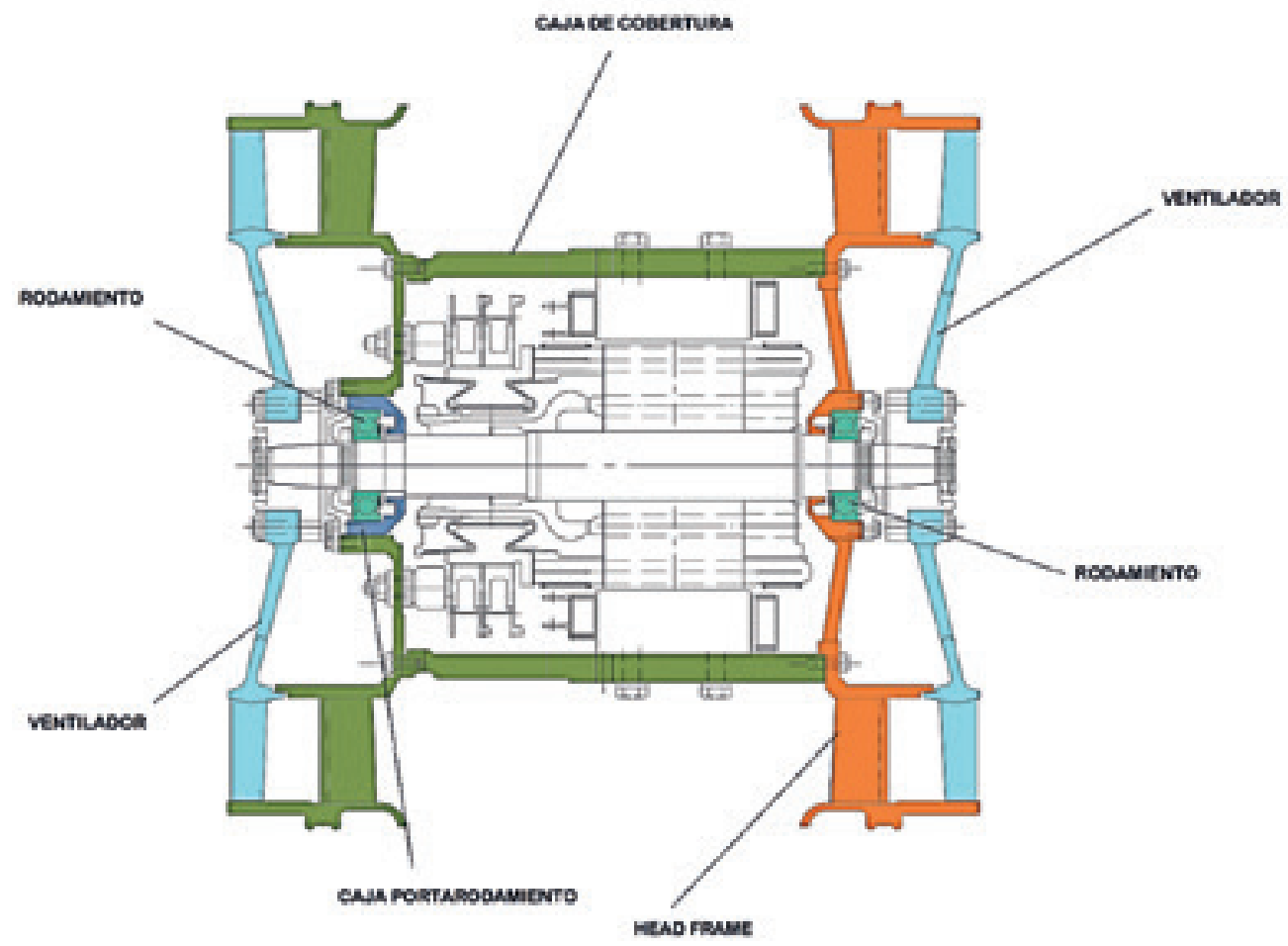

Fig. 4. Vista en corte del blower señalando sus partes mecánicas.

Fuente: Manual Blower Unit Model 5GY19 GEK-87940C.

De la revisión mecánica del blower se definió que se mantendrán los rodamientos del motor eléctrico original, por lo que, a partir de sus dimensiones, se diseña el diámetro del eje del motor hidráulico a implementar. La caja portarrodamientos se reutilizará sin presentar ninguna variación. El head frame de aluminio y acero en el núcleo no presentará cambios. Los ventiladores de aluminio de $30 \mathrm{~cm}$ de radio se mantendrán, debido a su eficiencia en el proceso. No presentan resistencia al giro y el vencimiento del torque se presenta a través de las pruebas con el dinamómetro, tal como se muestra en la figura 5 . 


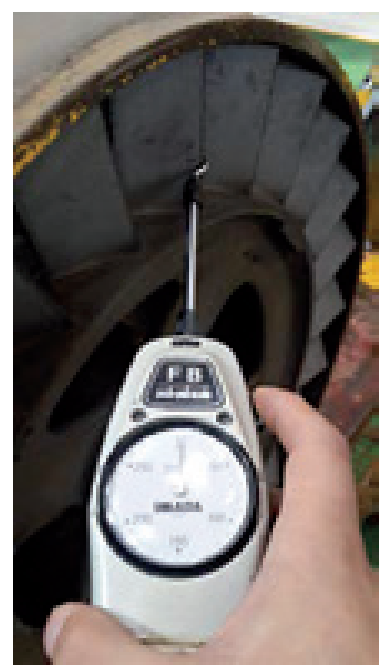

Fig. 5. Aplicación de fuerza

para vencer la inercia.

Fuente: elaboración propia.

La estructura eléctrica del blower consta de cinco partes: rotor, estator (bobinas), portacarbones, soporte aislante y cables de potencia (ver figura 6).

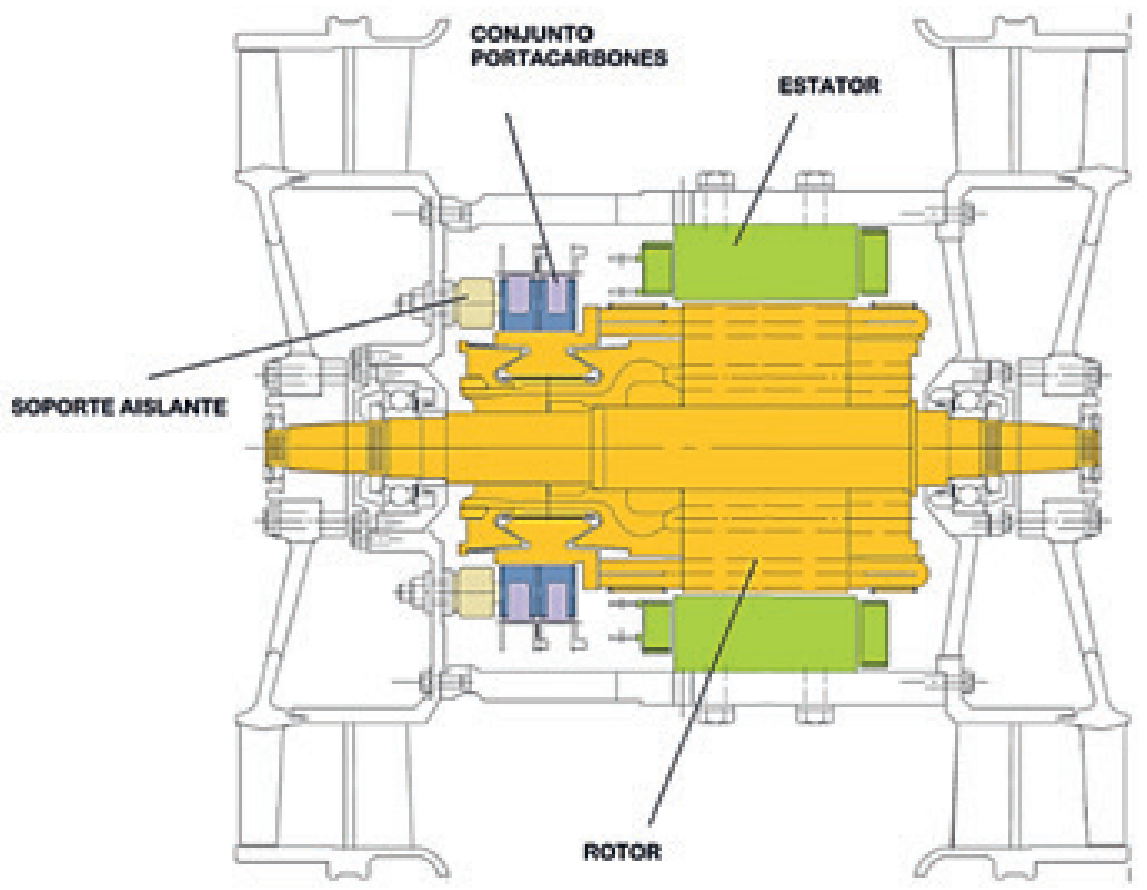

Fig. 6. Vista en corte del blower señalando sus partes eléctricas.

Fuente: Manual Blower Unit Model 5GY19 GEK-87940C.

En el desarrollo de la reingeniería, se plantea el reemplazo del sistema eléctrico por un sistema hidráulico. 


\subsection{Sistema hidráulico}

La modificación hidráulica se inicia a partir del reemplazo de los dos rotores de los blowers por un motor hidráulico, que es activado por una electroválvula que envía señal cuando el sistema de retardo está funcionando. De igual modo, el nuevo sistema cuenta con componentes de protección de acuerdo con el caudal y la presión de trabajo. Como punto fundamental, el sistema requiere de una bomba generadora del caudal necesario para poder ser activado, por lo que se utilizará la misma bomba del sistema de levante como fuente de energía hidráulica. Lo anterior es posible dado que el proceso de retardo y levante, por diseño de fábrica, no pueden funcionar simultáneamente, tal como se muestra en la figura 7 .

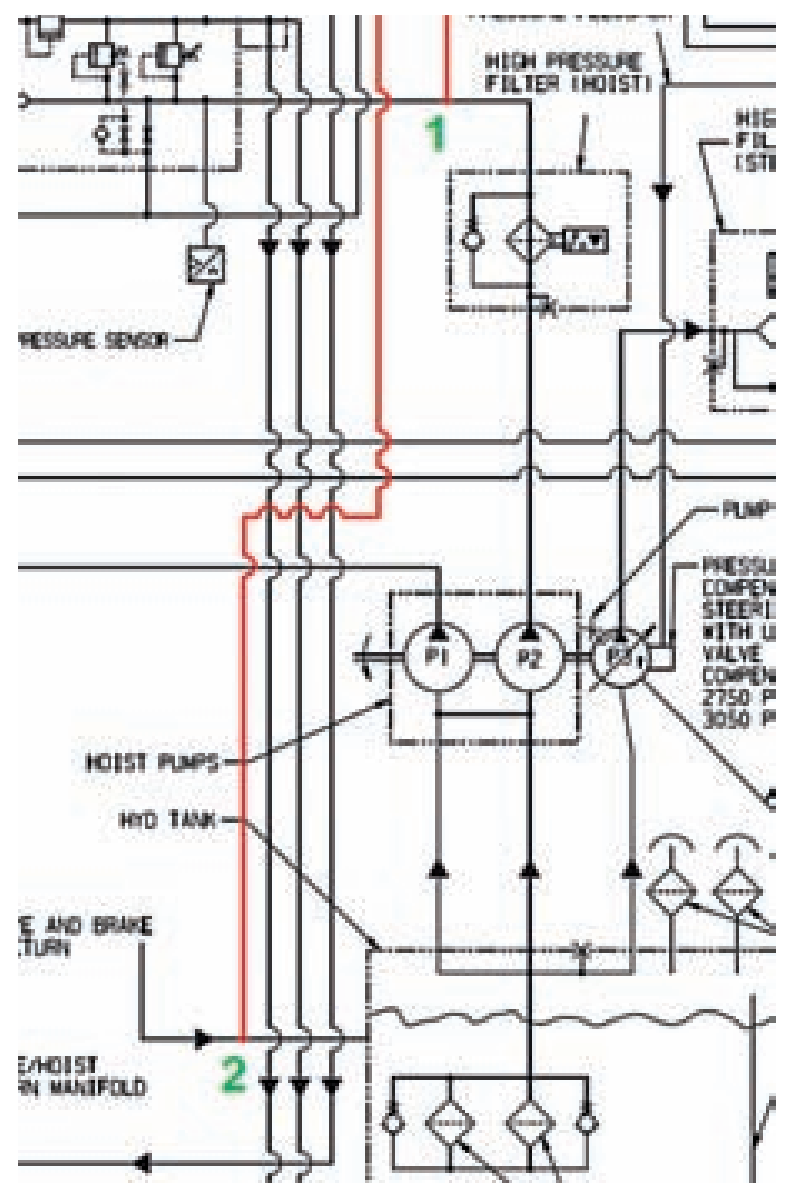

Fig. 7. Inicio del diseño desde la bomba de levante.

Fuente: Manual de Taller 930E-4SE CEBM020000.

El sistema hidráulico modificado parte desde la salida de la bomba de levante. La primera obstrucción del flujo empieza en la válvula reductora de caudal fija, la cual reduce el caudal a $1 / 5$ de su capacidad. Luego, este se reduce nuevamente hasta la capacidad necesaria para el correcto funcionamiento del motor hidráulico. Finalmente, el sistema cuenta con una electroválvula de accionamiento que impide y permite el paso del flujo de acuerdo con la señal que mande el sistema eléctrico. El flujo 
continúa por la válvula reguladora, que envía el caudal necesario al motor hidráulico, que es el actuador del sistema de ventilación. Finalmente, el flujo cae y pasa al tanque hidráulico, donde se cierra el circuito hidráulico. Todo el sistema modificado se muestra en la figura 8.

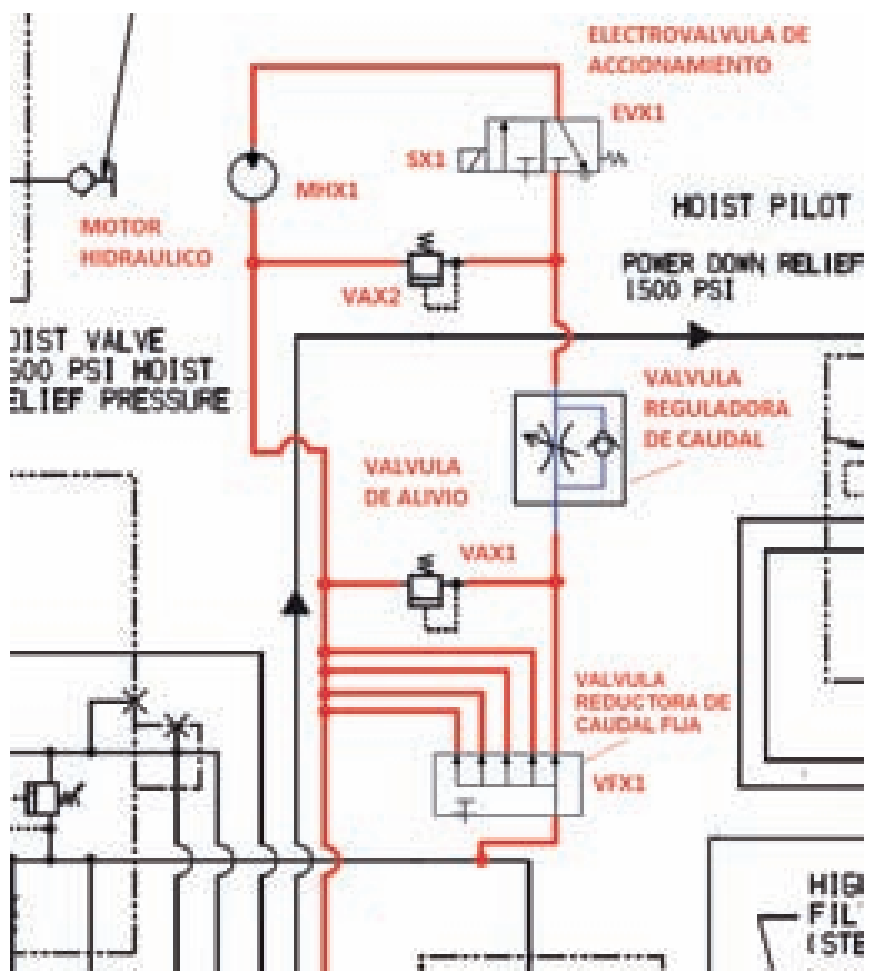

Fig. 8. Diseño del sistema hidráulico modificado.

Fuente: elaboración propia.

\subsection{Sistema eléctrico}

Luego de haber concluido la modificación hidráulica, se procede a realizar la evaluación del sistema eléctrico. Esta modificación está basada en la activación de la electroválvula (EVX1). Por lo tanto, la lógica de operación del nuevo sistema eléctrico consiste en que en cuanto el operador aplique el sistema de frenado de retardo, el sistema envía una señal eléctrica que coincidirá, en voltaje y potencia, con la entrada requerida en el solenoide de la electroválvula y, de este modo, permitirá el paso del caudal proveniente de la bomba hidráulica.

Como se ve en la figura 9, en las líneas del contactor RP1, se encuentran cinco resistencias de bajo ohmiaje colocadas en serie. El arreglo comienza al abrir el contactor RP1 y colocar una resistencia en serie RX1 y una en paralelo a esta última mencionada RX2 para poder manipular el solenoide que requiere la potencia de salida de la resistencia RX2. Las características de funcionamiento de la electroválvula fueron definidas en el sistema hidráulico con un voltaje de $24 \mathrm{~V}$ y una potencia de $51 \mathrm{~W}$. 


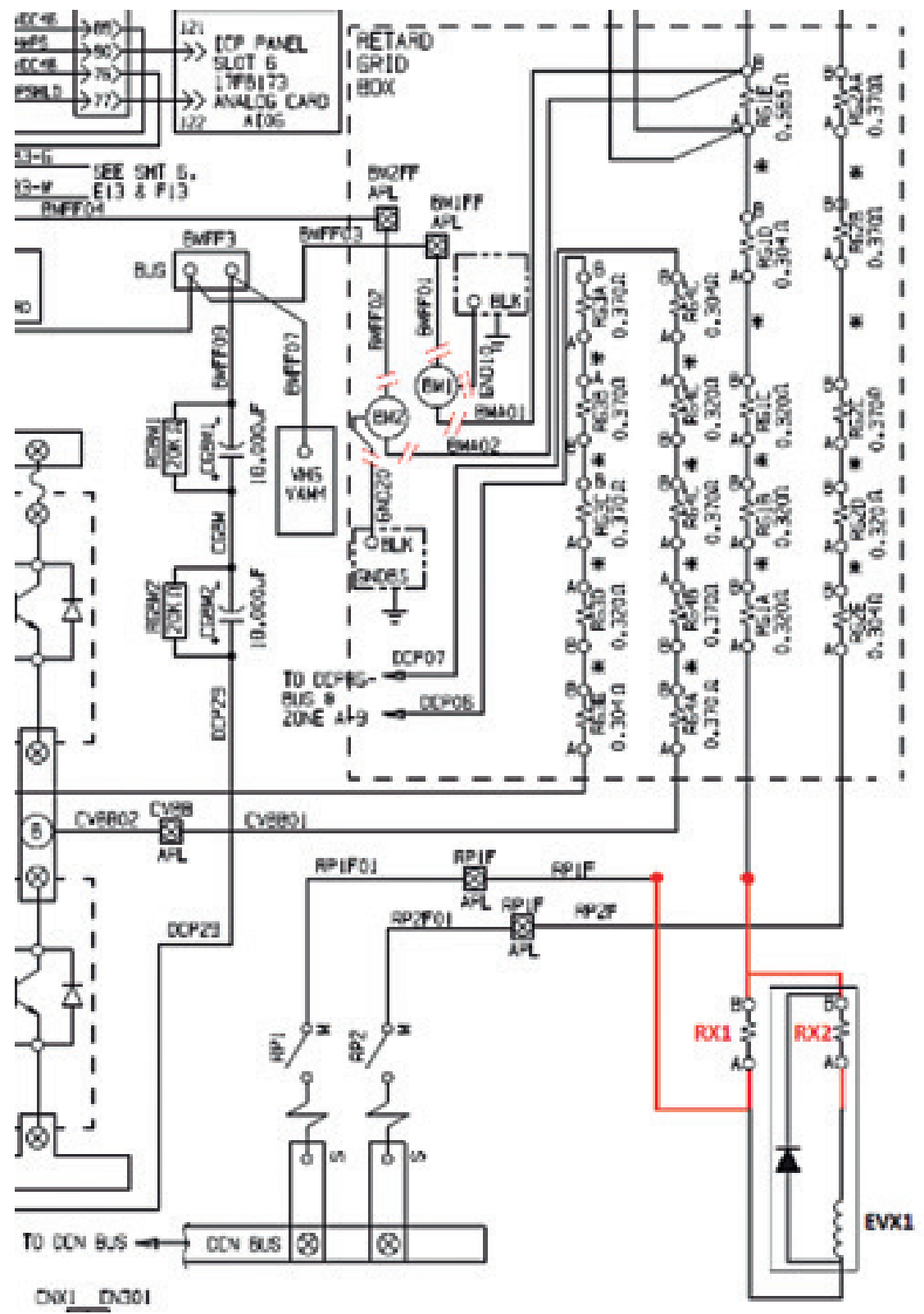

Fig. 9. Plano eléctrico modificado.

Fuente: Manual de Taller 930E-4SE CEBM020000.

\subsection{Sistema de transmisión mecánica}

Con las modificaciones hidráulicas del sistema, se realiza el diseño mecánico complementario para poder alcanzar los parámetros de velocidad requeridos por el sistema de blowers. Con este propósito, se decide implementar un sistema de poleas para la transmisión mecánica, las cuales se diseñan a 
partir de las condiciones dimensionales mecánicas vistas en el punto 2.1. La distribución de poleas se muestra en la figura 10.

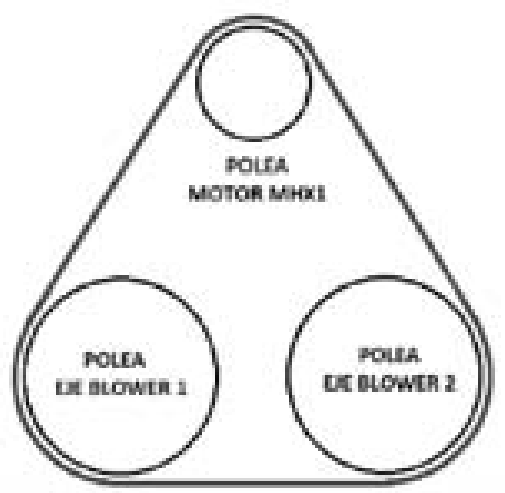

Fig. 10. Plano eléctrico modificado. Fuente: elaboración propia.

El diseño mecánico nos ofrece resultados para el diseño de las tres poleas. Se concluye que las dos poleas mayores ubicadas en los ejes de giro de los blowers tendrán dimensiones interiores de 54.03 mm y dimensiones exteriores de $113.40 \mathrm{~mm}$. La polea menor, ubicada en el eje del motor hidráulico, deberá tener un diámetro interior cónico con diámetro mayor de $0.687 \mathrm{~mm}$, altura de $0.854 \mathrm{~mm}$ y ángulo de generatriz de 1/8, mientras que su diámetro exterior será de $87.32 \mathrm{~mm}$. El diseño parte de las condiciones geométricas mostradas en la figura 11 .

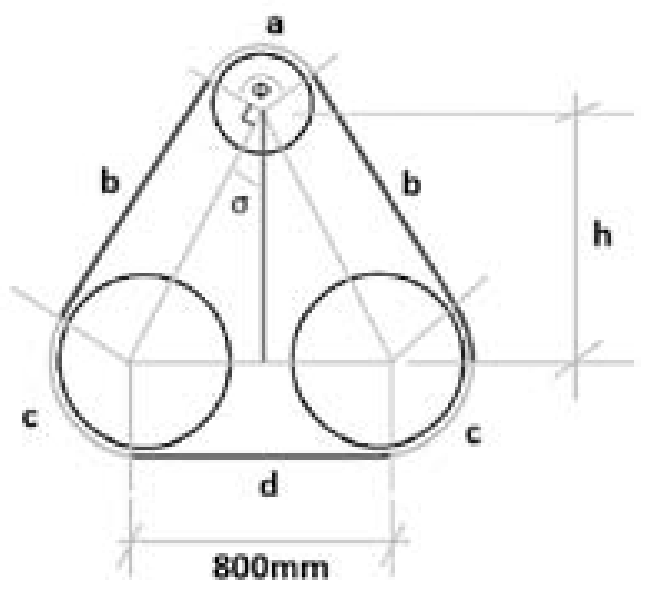

Fig. 11. Plano eléctrico modificado.

Fuente: elaboración propia.

Finalmente, a partir de diseños mecánicos de superficie de contacto de la faja con la polea del motor mayor a $160^{\circ}$, con corte trapezoidal como muestra la figura 12 , se obtiene un diseño de la correa Optibelt SK tipo SPZ de 5700, con un factor de seguridad de 11.48 . 


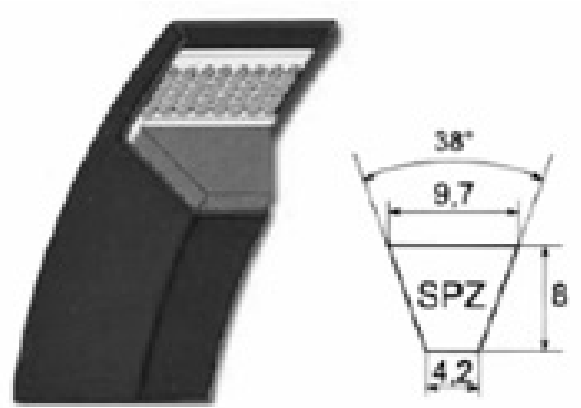

Fig. 12. Plano eléctrico modificado.

Fuente: http://www.gnuttibortolo.com/eng/scheda_articolo.jsp?idarticolo=48133

\subsection{Kit de repuestos}

Con los resultados de las modificaciones hidráulica, eléctrica y mecánica, se obtiene el kit de implementación del sistema, cuyos componentes se muestran en la tabla 1 .

TABLA 1. KIT DE IMPLEMENTACIÓN DEL SISTEMA DE FRENADO

\begin{tabular}{|c|c|c|}
\hline Pales & Cantilad & Unidales \\
\hline Mangueta de $1^{-}$ & 30 & m \\
\hline Mangueta de $1 / 2^{2}$ & 25 & m \\
\hline Menguena de $3 / 4^{2}$ & $\mathbf{s}$ & $\mathrm{m}$ \\
\hline Menpuera de $3 / 8^{\circ}$ & $\mathbf{5}$ & m \\
\hline Walvula reductora de cavdal tio & 1 & u \\
\hline Acoples de I" para valvila reductora de caudal tio & 6 & $\underline{u}$ \\
\hline Valwala de alivie de 20SOPSII & 1 & u \\
\hline Acoples de $1 / 3$ & $\frac{2}{2}$ & u \\
\hline Vivula reguladora de caudal & 1 & $u$ \\
\hline 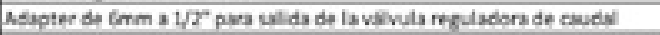 & 1 & $u$ \\
\hline Asapter de $1^{\prime \prime}$ a tome para la entrada de la villuvla reguladora de caudal & 1 & 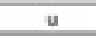 \\
\hline Vivela de divio de abour & 1 & u \\
\hline Acople de $X "$ para la vilvula de alinis de $1000 \mathrm{r}$ & 1 & u \\
\hline Acople de $1^{\prime \prime}$ para lavilinda de alivio de 100ar & 1 & $u$ \\
\hline Electrovsluvila de acolonamiento & 1 & u \\
\hline Acoples de $2 / Z^{\prime}$ para la electroval wula de acolonamiento & 2 & $\underline{4}$ \\
\hline 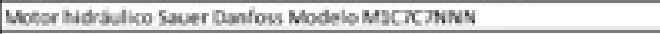 & 1 & u \\
\hline Altapter de $\mathrm{X}^{\prime \prime}$ a $12 \mathrm{~mm}$ para el motor hidralulice & 1 & 4 \\
\hline Asapter de $13 \mathrm{~mm}$ a $\mathrm{X}^{4}$ para el motor hidriclice & 1 & $u$ \\
\hline Acople tipo T $\left(I^{\prime}, 1^{n}, \mathrm{I}^{\prime \prime}\right)$ & 5 & $u$ \\
\hline 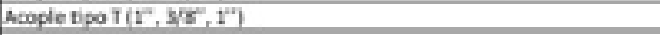 & 1 & 4 \\
\hline Acople tipo T(L/2, $\left.x^{20}, x^{2}\right)$ & 1 & u \\
\hline Acople tipo $\mathrm{T}\left(\mathrm{L} / \mathrm{Z}^{\prime \prime}, \mathrm{I}^{\prime \prime}, 1^{\prime \prime}\right)$ & 1 & u \\
\hline Acople tipo T $\left(\mathrm{r}^{r}, 3 / \mathrm{s}^{\prime}, \mathrm{Y}^{\prime}\right)$ & 1 & u \\
\hline Acople de 1" para instaladion de la manguera de I" al tanove hidravilico & 1 & $\underline{u}$ \\
\hline Polea para motor hidriculico & 1 & 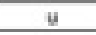 \\
\hline Pole a para eje de blower 1 & 1 & u \\
\hline Pole a para eje de blower? & 1 & $u$ \\
\hline fajas & 1 & $u$ \\
\hline Resistenois de a ósa & 1 & 4 \\
\hline Guiadores (40 de $1^{\prime \prime}, 20$ de $\mathrm{s}^{2 \prime}, 20$ de $1 / 8,20$ de $1 / 2$ ) & 100 & $u$ \\
\hline Cobles de potencia & 30 & $m$ \\
\hline Cosectores variados & 30 & $u$ \\
\hline Scocrse del motor & 1 & u \\
\hline Panes de instaladion y peccedimienso & 1 & u \\
\hline
\end{tabular}

Fuente: elaboración propia. 


\subsection{Simulación del sistema}

Luego de la obtención de la lógica de activación del nuevo sistema de ventilación de las parrillas a través de los blowers, se procede a diseñarlo para su verificación y comprobación virtual. En la figura 13, se observa la distribución general de la implementación del nuevo sistema de ventilación.

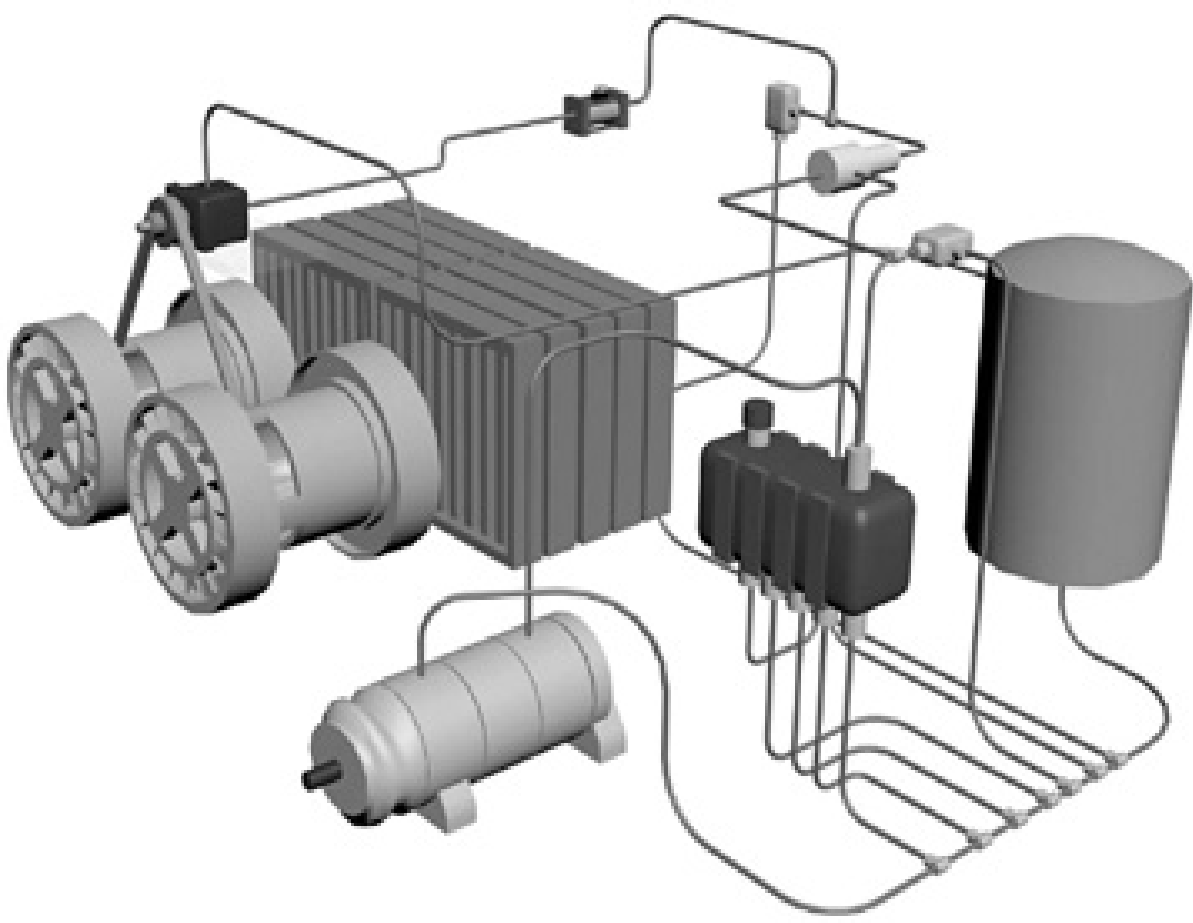

Fig. 13. Simulación del sistema de ventilación de parrillas.

Fuente: elaboración propia.

\section{Resultados: comparativa final del impacto económico}

La mina en la que se enfocó la investigación cuenta con 92 camiones y 5 palas hidráulicas, por lo que la criticidad de los equipos, así como cualquier falla de producto no prevista para ellos, se traduce en grandes impactos a nivel económico, comercial, social y también a nivel de seguridad.

Según el estudio de fallas realizado por la empresa Komatsu Mitsui Maquinarias Perú S.A. en base a las órdenes de trabajo efectuadas hasta el 2015, se concluye que el 100\% de las fallas prematuras de blower provenientes del Altiplano peruano son prematuras y se deben a causas referidas a desperfectos eléctricos, y se presentan, en promedio, a las 8036 horas, lo que equivale a un $31.14 \%$ de la vida regular del blower.

El impacto económico, utilizando el actual sistema de blowers con activación eléctrica, ha generado un costo total de 26.3 millones de dólares, lo que equivale a la suma del costo de mantenimiento del blower más el costo de impacto por equipo inoperativo. Por otro lado, la inversión total por el nuevo kit del sistema de frenado, que incluye su adquisición e instalación, es de alrededor de 7.1 millones de dólares, de lo que se obtiene una diferencia a favor de la minera de 19.1 millones de dólares, resultados que se muestran de manera resumida en la tabla 2. 
TABLA 2. RESUMEN DEL IMPACTO FINAL

\begin{tabular}{|c|c|c|}
\hline $\begin{array}{c}\text { Impacto económico con los } \\
\text { Blowers eléctrico }\end{array}$ & $\begin{array}{c}\text { Costo total de la modificación } \\
\text { con los Blowers hidráulicos }\end{array}$ & $\begin{array}{c}\text { Impacto económico favorable } \\
\text { con la modificación }\end{array}$ \\
\hline$\$ 26,303,560.96$ & $\$ 7,152,819.95$ & $\$ 19,150,741.01$ \\
\hline
\end{tabular}

Fuente: elaboración propia.

\section{Conclusiones}

De la presente investigación, se pueden extraer las siguientes conclusiones:

a) Fue posible rediseñar el actual sistema de frenado por retardo a través del cambio de actuadores eléctricos por sistemas robustos hidráulico-mecánicos, que modifican las líneas hidráulicas, bajo criterios de ingeniería hidráulica, lo que permitió proteger el sistema con la manipulación del caudal y control de presión hidráulico.

b) Se generó una señal para activar el solenoide que permite el paso del fluido hacia el motor hidráulico mediante un arreglo de resistencias eléctricas para el contactor RP1, lo que hizo factible la correcta activación del sistema hidráulico-mecánico.

c) Se redujo el impacto económico por fallas prematuras, ya que la implementación del kit de mejora y el cambio del sistema convencional eléctrico han dado como resultado una diferencia favorable de 19.15 millones de dólares.

\section{Observaciones y recomendaciones}

1. Se recomienda aplicar el nuevo kit en un primer camión minero piloto donde se verifiquen los cálculos teóricos alcanzados. Durante la implementación y pruebas, se deberá registrar, en cada una de las etapas, las observaciones y complicaciones.

2. Los camiones mineros cuentan con líneas eléctricas de potencia que alcanzan valores de 1,300 voltios, por lo que, durante su manipulación y pruebas, se deberá tener el cuidado respectivo al realizar las modificaciones eléctricas.

3. La electroválvula es el dispositivo que activa la modificación eléctrica e hidráulica. Por ello, su ubicación deberá estar aislada y protegida de agentes externos para evitar daños en su correcto funcionamiento.

4. El motor hidráulico utilizado deberá cambiarse anualmente debido a su bajo costo, lo que evitará retrasos por equipo parado.

\section{Referencias}

[1] Morales, J., Establecimiento de mejores prácticas para el control de contaminación del combustible y aceite en la flota de camiones eléctricos EH5000, Tesis Pre-Grado, Universidad de Pamplona, Colombia, 2007.

[2] Guerrero, N., Ingeniería e implementación de un prototipo parcial de una válvula de control de flujo, Tesis PreGrado, Universidad de Pamplona, Colombia, 2006.

[3] Navarro, R., Diseño de un controlador neurodifuso para la conducción autónoma de un camión minero de dos cuerpos, Tesis de Maestría, Pontificia Universidad Católica del Perú, Perú, 2013. 
[4] Quispe, H., Diseño del sistema de transmisión de potencia hidráulica de un alimentador de mineral de 150HP, Tesis Pre-Grado, Universidad Nacional de Ingeniería, Perú, 2008.

[5] Vidal, M., Estudio del cálculo de camiones para una operación minera a cielo abierto, Tesis Pre-Grado, Pontificia Universidad Católica del Perú, Perú, 2010.

[6] Baldeón, Z., Gestión en las operaciones de transporte y acarreo para el incremento de la productividad en CIA. Minera Condestable S.A., Tesis Pre-Grado, Pontificia Universidad Católica del Perú, Perú, 2011.

[7] Marchesi, O. \& Gaveglio, F. \& Salicetti, H. \& La Torre, O. \& Craig, D. \& Gravelle, J., 2013: Industria Minera Guía de Negocios en el Perú, Pricewaterhouse Coopers, Perú, 2013.

[8] Komatsu America Corp., Manual de taller 930E-4SE Camión Tolva CEBM02000, Komatsu, Estados Unidos, 2013.

[9] Anglo American, Curso Mantención de Camiones Komatsu 830E - 930E-3, Neutralis Capacitación, Chile, 2007.

[10] GE Transportation Systems. Dinamic retarding assembly 17EM136/17EM131, General Electric, Estados Unidos, 2003.

[11] GE Transportation Systems, Blower Unit Model 5GY19, General Electric, Estados Unidos, 2000. 
